\title{
Autism-linked gene SYNGAP1 molds synaptic plasticity, learning
}

\author{
BY ANGIE VOYLES ASKHAM
}

21 OCTOBER 2021

Even partial loss of the autism-linked gene SYNGAP1 impairs the brain's ability to respond to sensory experiences, according to a new study in mice. The finding may help to explain why people with SYNGAP1 mutations tend to have learning difficulties and a high tolerance for pain.

The SYNGAP1 protein abounds at excitatory synapses and shapes plasticity there, helping neighboring neurons to strengthen or weaken their connections. But it was previously unclear how SYNGAP1 affects 'ensembles,' groups of neurons with coordinated activity that are considered the "direct neural correlate of behavior and thought," says lead researcher Gavin Rumbaugh, professor of neuroscience at Scripps Research in Jupiter, Florida.

To encode new information about a sensory experience, a neuronal ensemble needs to redistribute its activity: Some neurons ramp up their chatter while others dial it down, keeping the group's overall activity the same. "It's learning, basically," Rumbaugh says.

That redistribution is defunct in mice that lack a copy of SYNGAP1, the new work shows. Only the decrease in activity happens, resulting in a weakened ensemble overall. But restoring typical SYNGAP1 expression levels in adult model mice normalizes the activity distribution, the team found.

"It really shows that SYNGAP1 is playing a role in the mature brain," says Kimberly Huber, professor of neuroscience at the University of Texas Southwestern Medical Center in Dallas, who was not involved in the work.

\section{New sensations:}

Rumbaugh and his colleagues used calcium imaging to record the ensembles that activate when mice receive a gentle touch to their whiskers. The neurons in those ensembles could be divided 


\section{Spectrum | Autism Research News}

https://www.spectrumnews.org

into three groups, the team found: More than half of the cells were weakly active during whisker stimulation, about 30 percent were moderately active, and about 7 percent were highly active. That activity pattern remained the same in both wildtype and SYNGAP1-deficient mice when the researchers recorded from the same neurons 13 days later.

"What that says is that these ensembles are basically stable when you don't give the animal a new experience," Rumbaugh says.

The team then trimmed all but one whisker on each mouse and returned the animals to their cages - a move Rumbaugh likens to numbing three fingers and a thumb on a person's hand and asking them to identify objects by rummaging their hand through a bag: The once-familiar experience would suddenly feel new.

As expected, the overall activity of the ensembles did not change in the wildtype mice, but the distribution of the ensemble's activity did: The weakly active cells scaled up their firing, the highly active cells dramatically suppressed their responses, and the moderately active cells showed little change.

Similarly, in the SYNGAP1 mice the highly active cells grew quieter, and the moderately active cells held steady. But the weakly active cells did not ramp up their signaling after the new experience, Rumbaugh and his colleagues found.

"That's the twist. And it has a complex effect on the circuit," says Richard Huganir, director of neuroscience at Johns Hopkins University in Baltimore, Maryland, who was not involved in the study.

Instead of the overall ensemble activity remaining stable in response to the new experience, the lack of SYNGAP1 weakens activity, which may explain why SYNGAP1 mice have poor whiskerdependent learning, as Rumbaugh and his colleagues previously reported.

\section{Learning curve:}

Wildtype mouse neurons that activate at the same time end up strengthening their synapse, further experiments in pairs of cultured cells showed; neurons that become active slightly out of sync weaken their connection.

By contrast, neurons from mice that lack a copy of SYNGAP1 weaken their connection with cells that fire out of sync but do not strengthen their connection with those that activate at the same time - suggesting that the gene is essential for this mechanism.

Wildtype mice also have additional presynaptic boutons - the nubs on a neuron that form synapses with other cells - after a new sensory experience, the team found using two-photon imaging. 


\section{Spectrum | Autism Research News}

https://www.spectrumnews.org

SYNGAP1-deficient mice instead respond with fewer presynaptic boutons.

"SYNGAP1 must be stimulating some mechanism that increases synaptic input into these networks, forming new synapses," Rumbaugh says. "We think that's why these low-active neurons can't scale up a whisker experience" in the SYNGAP1-deficient mice.

SYNGAP1 mice engineered so that the silenced copy of the gene can be reinstated in adulthood have ensemble activity that looks like that of wildtype mice, the team also found.

That suggests that the potential window for treatment of people with SYNGAP1 mutations extends beyond early development, Huganir says.

It also points to the possibility of harnessing SYNGAP1's ability to strengthen synapses and improve learning in other situations, Rumbaugh says. He and his colleagues are looking for compounds that can boost SYNGAP1 expression.

"We think they are going to be cognitive enhancers" that make ensemble plasticity more efficient overall, he says.

Cite this article: https://doi.org/10.53053/USKC1831 